\title{
Resumming perturbative series in the presence of monopole bubbling effects
}

\author{
Masazumi Honda ${ }^{1, *}$ and Daisuke Yokoyama ${ }^{2, \dagger}$ \\ ${ }^{1}$ Department of Particle Physics, Weizmann Institute of Science, Rehovot 7610001, Israel \\ ${ }^{2}$ Department of Mathematics, Kings College London, The Strand, London WC2R 2LS, United Kingdom, \\ and Department of Physics and Center for Field Theory and Particle Physics, Fudan University, \\ 220 Handan Road, 200433 Shanghai, China
}

(Received 14 March 2019; published 25 July 2019)

\begin{abstract}
The monopole bubbling effect is the screening of magnetic charges of singular Dirac monopoles by regular 't Hooft-Polyakov monopoles. We study the properties of a weak coupling perturbative series in the presence of monopole bubbling effects as well as instantons. For this purpose, we analyze supersymmetric 't Hooft loop in four-dimensional $\mathcal{N}=2$ supersymmetric gauge theories with Lagrangians and nonpositive beta functions. We show that the perturbative series of the 't Hooft loop is Borel summable along the positive real axis for fixed instanton numbers and screened magnetic charges. It turns out that the exact result of the 't Hooft loop is the same as the sum of the Borel resummations over instanton numbers and effective magnetic charges. We also obtain the same result for supersymmetric dyonic loops.
\end{abstract}

DOI: 10.1103/PhysRevD.100.025012

\section{INTRODUCTION}

Progress on quantum field theory (QFT) hinges on understanding nonperturbative effects such as instantons, monopoles, vortices, and so on. One of the much less understood nonperturbative effects is the monopole bubbling effect, which is the screening of magnetic charges of singular Dirac monopoles [1] by regular' $t$ Hooft-Polyakov monopoles $[2,3]$. The aim of this paper is to understand the properties of a weak coupling perturbative series in the presence of monopole bubbling effects as well as instantons.

The monopole bubbling effects typically appear in a 't Hooft loop [5], which is a magnetic version of a Wilson loop and detects the Higgs phase by an area law, while an area law of the Wilson loop implies confinement [6]. The 't Hooft loop can be defined as a partition function with singular boundary conditions for gauge fields given by Dirac monopoles [7]. For example, a straight 't Hooft line along the Euclidean time at spatial origin in $\mathbb{R}^{4}$ is described by

$$
F=\frac{B}{4} \epsilon_{\mu \nu \rho} \frac{x^{\mu}}{|x|^{3}} d x^{\nu} \wedge d x^{\rho},
$$

where $B$ is a flux on $S^{2}$ surrounding the loop $B=\frac{1}{2 \pi} \int_{S^{2}} F$. It is known that the 't Hooft loop receives not only instanton

*masazumi.hondaATweizmann.ac.il

"dyokoyamaATfudan.edu.cn

Published by the American Physical Society under the terms of the Creative Commons Attribution 4.0 International license. Further distribution of this work must maintain attribution to the author(s) and the published article's title, journal citation, and DOI. Funded by SCOAP ${ }^{3}$. corrections but also monopole bubbling effects. In this paper, we study perturbative series of half-Bogomolnyi-PrasadSommerfield 't Hooft loops in $4 \mathrm{~d} \mathcal{N}=2$ supersymmetric (SUSY) gauge theories. To preserve SUSY, we need an additional boundary condition for the adjoint scalar $\Phi$ in the $\mathcal{N}=2$ vector multiplet: $\Phi=\frac{B}{2|x|}$. Here we analyze the SUSY 't Hooft loops put on a curved space.

Before going to our setup in detail, let us recall the general expectation on a perturbative series in QFT. A perturbative series in QFT is typically not convergent [8]. In mathematics, there is a standard way to resum a nonconvergent series called Borel resummation. Given a perturbative series $\sum_{\ell=0}^{\infty} c_{\ell} g^{a+\ell}$ of a quantity $I(g)$, its Borel resummation along $\mathbb{R}_{+}$is defined by

$$
\mathcal{S}_{0} I(g)=\int_{0}^{\infty} d t e^{-(t / g)} \mathcal{B} I(t),
$$

where $\mathcal{B} I(t)$ is an analytic continuation of the formal Borel transformation $\sum_{\ell=0}^{\infty} \frac{c_{\ell}}{\Gamma(a+\ell)} t^{a+\ell-1}$ after the summation. A perturbative series in typical QFT is expected to be non-Borel summable due to singularities along $\mathbb{R}_{+}$of $\mathcal{B} I(t)$ and the Borel resummation formula has ambiguities, since the integral depends on how to avoid the singularities. However, it is unclear, in general, when perturbative series in QFT are Borel summable. Another important question is, in the case that we do not have Borel ambiguities, how the resummation is related to exact results.

In Ref. [9], one of the authors initiated to address these questions. It has been proven that perturbative series in $4 \mathrm{~d} \mathcal{N}=2$ and $5 \mathrm{~d} \mathcal{N}=1$ SUSY gauge theories with Lagrangians are Borel summable along $\mathbb{R}_{+}$for various 
observables in sectors with fixed instanton numbers [10]. This result for the $4 \mathrm{~d} \mathcal{N}=2$ case is expected from a recent proposal [13] on a semiclassical realization of IR renormalons [14], where the semiclassical solution does not exist in the $4 \mathrm{~d} \mathcal{N}=2$ theories [15] (see also [16]). In this paper, we study the 't Hooft loop in the $4 \mathrm{~d} \mathcal{N}=2$ theories, which receives monopole bubbling effects in addition to instanton effects.

Now we explain the details of our setup. Let us consider $4 \mathrm{~d} \mathcal{N}=2$ theories with Lagrangians and nonpositive beta functions. In this class of theories, we study the supersymmetric 't Hooft loop placed at a circle in a squashed sphere $S_{b}^{4}$, which is defined as the hypersurface in $\mathbb{R}^{5}$ :

$$
X_{0}^{2}+b^{-2}\left(X_{1}^{2}+X_{2}^{2}\right)+b^{2}\left(X_{3}^{2}+X_{4}^{2}\right)=r^{2} .
$$

In this geometry, we can place half-Bogomolnyi-PrasadSommerfield line operators on the circles $S_{b}^{1}$ and $S_{b^{-1}}^{1}$, which are given by $X_{3}=X_{4}=0$ and $X_{1}=X_{2}=0$ at fixed $X_{0}$, respectively. Although we explicitly discuss the case for $S_{b}^{1}$ at $X_{0}=0$, the result for $S_{b^{-1}}^{1}$ is simply obtained by the replacement $b \rightarrow b^{-1}$, which does not change our interpretation on the results.

The 't Hooft loop receives both instantons and monopole bubbling effects $[17,18]$ :

$$
T(B)=\sum_{k, \bar{k}, v} e^{-k S_{\text {inst }}-\bar{k} \bar{S}_{\text {inst }}-\operatorname{tr}\left(v^{2}\right) S_{\text {mono }}} T^{(k, \bar{k}, v)}(g, \theta),
$$

where $v$ denotes a screened monopole charge and

$$
S_{\text {inst }}=-2 \pi i \tau, \quad \bar{S}_{\text {inst }}=2 \pi i \bar{\tau}, \quad S_{\text {mono }}=-\frac{\pi b^{2}}{2 g},
$$

with a complex gauge coupling $\tau=\theta / 2 \pi+i / g$. Equation (4) is schematic in the sense that theories with a product gauge group have multiple couplings and multiple $(k, \bar{k}, v)$. Here we are interested in weak coupling expansion by (the square of) Yang-Mills coupling $g$ :

$$
T^{(k, \bar{k}, v)}(g, \theta) \simeq \sum_{\ell} c_{\ell}^{(k, \bar{k}, v)}(\theta) g^{\sharp+\ell},
$$

where $\sharp$ is a leading-order exponent. In this paper, we show that the perturbative series in the sector with fixed $(k, \bar{k}, v)$ is Borel summable along $\mathbb{R}_{+}[19]$ in the case that explicit expressions for instantons and monopole bubbling effects in the SUSY localization formula [20] are available. Our main result is

$$
T^{(k, \bar{k}, v)}(g, \theta)=\mathcal{S}_{0} T^{(k, \bar{k}, v)}(g, \theta) .
$$

Thus, we can rewrite the whole exact result in terms of the Borel resummation:

$$
T(B)=\sum_{k, \bar{k}, v} e^{-k S_{\text {inst }}-\bar{k} \bar{S}_{\text {inst }}-\operatorname{tr}\left(v^{2}\right) S_{\text {mono }}} \mathcal{S}_{0} T^{(k, \bar{k}, v)}(g, \theta) .
$$

This equation can be understood as "semiclassical decoding" [21] of the 't Hooft loop in $4 \mathrm{~d} \mathcal{N}=2$ theories, though the resurgence structure itself is trivial for this case.

\section{II. 'T HOOFT LOOP AND BOREL RESUMMATION}

\section{A. Exact result}

Instead of a path integral expression, we use a conjectural finite-dimensional integral representation for the 't Hooft loop [22-24] which has passed various nontrivial tests. There are exact results for the 't Hooft loop in the $4 \mathrm{~d} \mathcal{N}=2$ theories on round $S^{4}$ [24], $S^{1} \times \mathbb{R}^{3}$ [22], and $S^{1} \times S^{3}$ [25] by SUSY localization [20]. Although there is no explicit computation for our $S_{b}^{4}$ case, one can find the reasonable expression for the exact result by combining the results for round $S^{4}$ [24], $S^{1} \times \mathbb{R}^{3}$ [22], and the partition function on $S_{b}^{4}$ [26] (see also [27]). The expression is consistent with the exact results on round $S^{4}$ for $b=1$, the partition function on $S_{b}^{4}$ for $B=0$, and the Alday-GaiottoTachikawa relation [28] for some specific theories.

Let us consider the $4 \mathrm{~d} \mathcal{N}=2$ SUSY gauge theories with a semisimple gauge group $G=G_{1} \times \cdots \times G_{n}$, which are coupled to hypermultiplets of representations $\left(\mathbf{R}_{1}, \ldots, \mathbf{R}_{\mathbf{N}_{\mathbf{f}}}\right)$. According to Refs. [22-24], the 't Hooft loop is given by [29]

$$
T(B)=\sum_{v} \int d^{|G|} a Z_{\mathrm{cl}}^{(v)} Z_{1 \text { loop }}^{(v)} Z_{\mathrm{NP}}^{(v)}
$$

where $v$ describes the screened magnetic charges. The classical contribution $Z_{\mathrm{cl}}^{(v)}(a)$ is

$$
Z_{\mathrm{cl}}^{(v)}(a)=\prod_{p=1}^{n} \exp \left[-\frac{\operatorname{tr}_{p} a^{2}}{g_{p}}-b \theta_{p} \operatorname{tr}_{p}(a \cdot v)\right],
$$

where $g_{p}$ is proportional to the square of the one-loop effective Yang-Mills coupling at scale $1 / r$ and $\operatorname{tr}_{p}$ denotes trace in the group $G_{p} \subset G$. The one-loop determinant $Z_{1 \text { loop }}^{(v)}$ has contributions from both the poles and the equator of $S_{b}^{4}$ [30]:

$$
\begin{aligned}
Z_{1 \text { loop }}^{(v)}(a) & =\left|Z_{N}\left(a_{N}\right)\right|^{2} Z_{\mathrm{eq}}(a), \\
Z_{N}(a) & =\left[\frac{\prod_{\alpha \in \Delta_{+}} \Upsilon(i \alpha \cdot a) \Upsilon(-i \alpha \cdot a)}{\prod_{I=1}^{N_{f}} \prod_{\rho_{I} \in \mathbf{R}_{\mathrm{m}}} \Upsilon\left(i \rho_{I} \cdot a+\frac{Q}{2}\right)}\right]^{1 / 2}, \\
Z_{\mathrm{eq}}(a) & =\frac{\prod_{I, \rho_{I}} \prod_{k=0}^{\left|\rho_{I} \cdot v\right|-1} \cosh ^{\frac{1}{2}}\left[\pi b\left(\rho_{I} \cdot a+i b\left(k-\frac{\left|\rho_{I} \cdot v\right|-1}{2}\right)\right)\right]}{\prod_{\alpha \in \Delta} \prod_{k=0}^{|\alpha \cdot v|-1} \sinh ^{\frac{1}{2}}\left[\pi b\left(\alpha \cdot a+i b\left(k-\frac{|\alpha \cdot v|}{2}\right)\right)\right]},
\end{aligned}
$$


where $Q=b+b^{-1}, a_{N}=a+\frac{i b v}{2}$, and $\Upsilon(x)$ is the Upsilon function defined by

$$
\Upsilon(x)=\prod_{m_{1}, m_{2} \geq 0}\left(m_{1} b+m_{2} b^{-1}+x\right)\left(m_{1} b+m_{2} b^{-1}+Q-x\right) .
$$

$Z_{\mathrm{NP}}^{(v)}$ expresses nonperturbative effects, which are more involved:

$$
\begin{aligned}
Z_{\mathrm{NP}}^{(v)}(a) & =Z_{\text {inst }}^{(v)}(a) Z_{\text {mono }}^{(v)}(a), \\
Z_{\text {inst }}^{(v)} & =\left|Z_{\text {Nek }}^{(v)}\left(a_{N}\right)\right|^{2}=\sum_{k, \bar{k}} e^{-k S_{\text {inst }}-\bar{k} \bar{S}_{\text {inst }}} Z_{\text {inst }}^{(k, \bar{k}, v)},
\end{aligned}
$$

where $Z_{\mathrm{Nek}}^{(v)}$ is the Nekrasov instanton partition function [32] with $\Omega$-background parameters $\left(\epsilon_{1}, \epsilon_{2}\right)=\left(b, b^{-1}\right)$ and $Z_{\text {mono }}^{(v)}$ is a contribution from monopole bubbling effects. Note that $Z_{\text {mono }}^{(v)}(a)$ for $v=B$ is trivial:

$$
Z_{\text {mono }}^{(B)}(a)=1,
$$

which reflects the absence of the monopole screening. Now we are interested in the properties of small- $g$ expansion of the object

$$
T^{(k, \bar{k}, v)}(g, \theta)=\int_{-\infty}^{\infty} d^{|G|} a Z_{\mathrm{cl}}^{(v)} Z_{1 \text { loop }}^{(v)} Z_{\mathrm{NP}}^{(k, \bar{k}, v)},
$$

where

$$
Z_{\mathrm{NP}}^{(k, \bar{k}, v)}(a)=Z_{\mathrm{inst}}^{(k, \bar{k}, v)}(a) Z_{\mathrm{mono}}^{(v)}(a)
$$

\section{B. $S U(N)$ superconformal QCD}

We first discuss a $4 \mathrm{~d} \mathcal{N}=2 S U(N)$ superconformal QCD for simplicity of explanations, which is SUSY QCD (SQCD) with $2 N$ fundamental hypermultiplets. We will consider more general theories later. The 't Hooft loop of our SQCD in the sector $(k, \bar{k}, v)$ is

$$
\begin{aligned}
& T_{\mathrm{SQCD}}^{(k, \bar{k}, v)}(g, \theta) \\
& =\int d^{N} a \delta\left(\sum_{j} a_{j}\right) e^{-(1 / g) \sum_{j} a_{j}^{2}-b \theta \sum_{j} v_{j} a_{j}} \\
& \quad \times \prod_{1 \leq i<j \leq N} \frac{\left|\Upsilon\left(i a_{N, i j}\right) \Upsilon\left(-i a_{N, i j}\right)\right|}{\prod_{k=0}^{\left|v_{i j}\right|-1}\left|\sinh \left[\pi b\left(a_{i j}+i b\left(k-\frac{\left|v_{i j}\right|}{2}\right)\right)\right]\right|} \\
& \quad \times \prod_{j=1}^{N} \frac{\prod_{k=0}^{\left|v_{j}\right|-1} \cosh ^{N}\left[\pi b\left(a_{j}+i b\left(k-\frac{\left|v_{j}\right|-1}{2}\right)\right)\right]}{\left|\Upsilon\left(i a_{N, j}+\frac{Q}{2}\right)\right|^{2 N}} Z_{\mathrm{NP}}^{(k, \bar{k}, v),}
\end{aligned}
$$

where $a_{i j}=a_{i}-a_{j}$ and $v_{i j}=v_{i}-v_{j}$. Let us study small- $g$ expansion of this object. Instead of explicitly computing perturbative coefficients, we explicitly find Borel transformation, somehow already hidden in Eq. (17) as in Refs. $[9,33]$. To see this, first we make a change of the variables as $a_{j}=\sqrt{t} \hat{x}_{j}$, where $\hat{\mathbf{x}}=\left(\hat{x}_{1}, \ldots, \hat{x}_{N}\right)$ is the unit vector in $\mathbb{R}^{N}$. Then, we rewrite the 't Hooft loop as

$$
T_{\mathrm{SQCD}}^{(k, \bar{k}, v)}(g, \theta)=\int_{0}^{\infty} d t e^{-(t / g)} f^{(k, \bar{k}, v)}(t, \theta),
$$

where

$$
\begin{aligned}
f^{(k, \bar{k}, v)}(t) & =t^{\sharp} \int_{S^{N-1}} d^{N-1} \hat{x} \delta\left(\sum_{j} \hat{x}_{j}\right) h^{(k, \bar{k}, v)}(t, \hat{x}), \\
h^{(k, \bar{k}, v)}(t, \hat{x}) & =\left.e^{-b \theta \sum_{j} v_{j} a_{j}} Z_{1 \text { loop }}^{(v)} Z_{\mathrm{NP}}^{(k, \bar{k}, v)}\right|_{a_{j}=\sqrt{t} \hat{x}_{j}} .
\end{aligned}
$$

The exponent $\sharp$ is a constant depending on $N$ and $v_{i j}$, whose detail is not important for our purpose [34]. The functions $f^{(k, \bar{k}, v)}$ and $h^{(k, \bar{k}, v)}$ depend on $\theta$, but we do not often write $\theta$ explicitly in the arguments for simplicity. Since the expression (18) is the form of the Laplace transformation and similar to the Borel resummation (2), one may wonder whether the function $f^{(k, \bar{k}, v)}(t)$ of the perturbative series is the Borel transformation. Indeed, we can prove that this is the case as in Refs. $[9,33]$ and the function $f^{(k, \bar{k}, v)}(t)$ itself is the Borel transformation of the perturbative series:

$$
f^{(k, \bar{k}, v)}(t, \theta)=\mathcal{B} T_{\mathrm{SQCD}}^{(k, \bar{k}, v)}(t, \theta) .
$$

The proof takes the following three steps. (i) We show that the integrand $h^{(k, \bar{k}, v)}(t, \hat{x})$ is identical to analytic continuation of a convergent power series of $t$. (ii) We exchange the order of the power series expansion of $h^{(k, \bar{k}, v)}(t, \hat{x})$ by $t$ and integration over $\hat{x}$. This is rigorously justified by proving uniform convergence of the small- $t$ expansion as a sufficient condition. (iii) It is easily seen that the coefficient of the perturbative series of $f^{(k, \bar{k}, v)}(t)$ is given by $c_{\ell}^{(k)} / \Gamma\left(\frac{\sharp+\ell}{2}\right)$ as guaranteed by the Laplace transformation (18).

First, let us focus on the perturbative sector $(k, \bar{k}, v)=$ $(0,0, B)$. We will consider nonperturbative effects later. To prove the uniform convergence of the small- $t$ expansion of $h^{(k, k, v)}(t, \hat{x})$, it is convenient to use the Weierstrass M test; i.e., we find a sequence $\left\{M_{\ell}\right\}$ such that $\left|h_{\ell}^{(0)}(\hat{x})\right|<M_{\ell}$ and $\sum_{\ell=0}^{\infty} M_{\ell} t^{\sharp+\ell}<\infty$ for fixed $t$. We can easily find such a series as in Refs. $[9,33]$ by replacing all the sources of negative contributions to the small- $t$ expansion by positive definite larger values [35]. As an explicit example, the following function generates $M_{\ell}$ :

$$
\frac{P(t) e^{b \theta \sqrt{t} \sum_{j}\left|v_{j}\right|-4\left(5 N^{2}-4 N\right) b^{2} t}}{\left(1-b^{2} t\right)^{4 N^{2}} \prod_{ \pm}\left(1-2 b^{2}(2 t \pm \sqrt{t} Q)\right)^{2\left(N^{2}-N\right)}},
$$


where $P(t)$ is an appropriate finite-order polynomial. Thus, $f^{(0,0, B)}(t)$ is actually the Borel transformation.

Now we can explicitly study analytic properties of the Borel transformation. Since we have expressed the Borel transformation in terms of the one-loop determinant $Z_{1 \text { loop}}^{(v)}$, this problem boils down to analytic properties of the oneloop determinant, whose details are studied in Appendix A for the general case. As a result, the one-loop determinant in our SQCD has degree- $2 N$ poles at

$$
a_{j}= \pm i\left[\left(m_{1}+\frac{\left|v_{j}\right|+1}{2}\right) b+\left(m_{2}+\frac{1}{2}\right) b^{-1}\right]
$$

where $m_{1}, m_{2} \in \mathbb{Z}_{\geq 0}$. We emphasize that all the apparent branch cuts are canceled in the whole expression. The most important point here is that the one-loop determinant does not have singularities for $a \in \mathbb{R}$. Thus, the Borel transformation has singularities (poles) only along $\mathbb{R}_{-}$for $b \in \mathbb{R}$ and the perturbative series is Borel summable along $\mathbb{R}_{+}$.

\section{General $\mathcal{N}=2$ theories with a Lagrangian}

We can easily generalize the above analysis to general $\mathcal{N}=2$ theories with a Lagrangian and nonpositive $\beta$ function. Taking the polar coordinate $a_{i}^{(p)}=\sqrt{t_{p}} \hat{x}_{i}^{(p)}$ with $\hat{x}_{i}^{(p)} \in S^{\left|G_{p}\right|-1}$, we find [36]

$$
T^{(k, \bar{k}, v)}(g, \theta)=\int_{0}^{\infty} d^{n} t e^{-\sum_{p=1}^{n}\left(t_{p} / g_{p}\right)} f^{(k, \bar{k}, v)}(t),
$$

where [37]

$$
\begin{aligned}
f^{(k, \bar{k}, v)}(t) & =t^{\sharp} \int_{\text {sphere }} d \hat{x} h^{(k, \bar{k}, v)}(t, \hat{x}), \\
h^{(k, \bar{k}, v)}(t, \hat{x}) & =\left.e^{-b \sum_{p} \theta_{p} \operatorname{tr}_{p} v \cdot a} Z_{1 \text { loop }}^{(v)} Z_{\mathrm{NP}}^{(k, \bar{k}, v)}\right|_{a_{i}^{(p)}={\sqrt{t_{p}} \hat{x}_{i}^{(p)}},}
\end{aligned}
$$

where $t^{\sharp}=\prod_{p=1}^{n} t_{p}^{\sharp_{p}}$ with a constant $\sharp_{p}$ unimportant for our purpose [38]. Again, Eq. (23) is similar to the form of Borel resummation. Indeed, one can show that the function $f^{(k, \bar{k}, v)}(t)$ is nothing but the Borel transformation of the perturbative series. Let us focus on the sector with $(k, \bar{k}, v)=(0,0, B)$ again. As in the previous case, we can always show that $f^{(k, \bar{k}, v)}(t)$ is the Borel transformation:

$$
\mathcal{B} T^{(\{k, \bar{k}, v\})}(t, \theta)=f^{(\{k, \bar{k}, v\})}(t, \theta) .
$$

Let us look at analytic properties of the Borel transformation. According to Appendix A, the one-loop determinant has singularities at

$\rho \cdot a= \pm i\left[\left(m_{1}+\frac{|\rho \cdot v|+1}{2}\right) b+\left(m_{2}+\frac{1}{2}\right) b^{-1}\right]$.
This shows that the one-loop determinant does not have singularities for real $a$ and, therefore, the perturbative series is Borel summable along the positive real axis.

\section{Instanton corrections}

Generalization to the nonzero instanton sector is also straightforward, because $Z_{\mathrm{Nek}}(a)$ with a fixed instanton number and omega background parameters $\left(\epsilon_{1}, \epsilon_{2}\right)=$ $\left(b, b^{-1}\right)$ is a rational function, which does not have poles for real $a$ unless $m_{1} b+m_{2} b^{-1}\left(m_{1,2} \in \mathbb{Z}\right)$ is purely imaginary. Therefore, Eq. (25) still holds in nonzero instanton sectors.

For example, the Nekrasov partition function for $U(N)$ SQCD with $N_{f}$ fundamental hypers is given by

$$
Z_{\mathrm{Nek}}(a)=\sum_{Y} e^{-|Y| S_{\text {inst }}} \frac{\left(\prod_{j=1}^{N} n_{j}^{f}(a, Y)\right)^{N_{f}}}{\prod_{i, j=1}^{N} n_{i, j}^{V}(a, Y)},
$$

where $Y=\left(Y_{1}, \ldots, Y_{N}\right)$ denotes a set of Young tableau in $U(N),|Y|$ is the total number of boxes of $Y$, and

$$
\begin{aligned}
n_{i, j}^{V}(a, Y) & =\prod_{s \in Y_{i}} E_{i j}(a, s)\left(Q-E_{i j}(a, s)\right), \\
E_{i j}(a, s) & =-b A_{Y_{j}}(s)+b^{-1}\left(L_{Y_{i}}(s)+1\right)-i a_{i j}, \\
n_{j}^{f}(a, Y) & =\prod_{s \in Y_{j}} \phi_{j}(a, s)\left(\phi_{j}(a, s)+Q\right), \\
\phi_{j}(a, s) & =-i a_{j}+b\left(s_{h}-1\right)+b^{-1}\left(s_{v}-1\right) .
\end{aligned}
$$

The indices $s=\left(s_{h}, s_{v}\right)$ label a box in Young diagram at the $s_{h}$ th column and $s_{v}$ th row, and $L_{Y}(s)\left[A_{Y}(s)\right]$ is the leg (arm) length of Young tableau $Y$ at $s$. The vector contribution gives poles at

$$
a_{i j}=-i b\left(\frac{v_{i j}}{2}-A_{Y_{j}}(s)\right)-i b^{-1}\left(L_{Y_{i}}(s)+1\right)
$$

and

$$
a_{i j}=i b\left(1-\frac{v_{i j}}{2}+A_{Y_{j}}(s)\right)-i b^{-1} L_{Y_{i}}(s) .
$$

Although the contribution from each Young diagram may have poles at $a_{i j}=0$, this type of pole is canceled after summing over Young diagrams with the same instanton number [20]. Thus, the Borel transformation has singularities only along $\mathbb{R}_{-}$and especially a perturbative series is Borel summable along $\mathbb{R}_{+}$even if we include the instanton corrections.

\section{E. Monopole bubbling effects}

Now let us add monopole bubbling effects. As in the instanton corrections, generalization is straightforward as 
long as we know explicit expressions of $Z_{\text {mono }}^{(v)}(a)$, because the monopole bubbling effect is described by a ratio of finite products of hyperbolic functions, whose poles are not on the real axis. Hence, Eq. (25) still holds in the presence of the monopole bubbling effects.

For example, for $S U(N)$ or $U(N)$ gauge theory with fundamentals and adjoints, the contribution from the monopole bubbling effects is given by

$$
Z_{\text {mono }}^{(v)}(a)=\sum_{Y} Z_{Y}^{\mathrm{vec}}(a) \prod_{I} Z_{Y}^{R_{I}}(a),
$$

where

$$
\begin{aligned}
Z_{Y}^{\mathrm{vec}}(a) & =\frac{1}{\hat{\prod}_{i, j, s \in Y_{i}, \pm} \sinh \frac{2 \pi b a_{i j}+i \pi b^{2}\left(A_{Y_{i}}(s)-L_{Y_{j}}(s) \pm 1\right)}{2}} \\
Z_{Y}^{\mathrm{adj}}(a) & =\hat{\prod}_{i, j, s \in Y_{i}} \cosh ^{2} \frac{2 \pi b a_{i j}+i \pi b^{2}\left(A_{Y_{i}}(s)-L_{Y_{j}}(s)\right)}{2} \\
Z_{Y}^{\mathrm{fund}}(a) & =\hat{\prod}_{j, s \in Y_{j}} \cosh \frac{2 \pi b a_{j}+i \pi b^{2}\left(s_{v}+s_{h}-1\right)}{2}
\end{aligned}
$$

The sum in Eq. (31) is over a set of Young tableau with the total number of boxes $\frac{1}{2} \operatorname{tr}\left(B^{2}-v^{2}\right)$. Note also that we have put the symbol ^for the sum and product, which means the sum over $Y$ and product over $s$ are constrained. Namely, we include only $Y$ and $s$ satisfying the constraints in contrast to the instanton corrections. We explain the details on this in Appendix B, because the constraints are quite complicated, and, nevertheless, their details are not so important for our purpose.

The most important thing for us is a singularity structure of $Z_{\text {mono }}^{(v)}(a)$. For a given single set of Young diagram $Y$, the vector contribution gives poles at

$$
a_{i j}=i b^{-1} m-\frac{i b}{2}\left(A_{Y_{i}}(s)-L_{Y_{j}}(s) \pm 1\right) .
$$

When $A_{Y_{i}}(s)-L_{Y_{j}}(s) \pm 1 \neq 0$ or $m \neq 0$, the poles are not located along the real axis unless $b^{2}$ is purely imaginary. When $A_{Y_{i}}(s)-L_{Y_{j}}(s) \pm 1=0$, we have apparent poles at $a_{i j}=0$, but this type of pole is finally canceled from other Young diagrams as long as we consider well-defined 't Hooft loops. Thus, $Z_{\text {mono }}^{(v)}(a)$ does not have singularities along the real axis, and the perturbative series is Borel summable along $\mathbb{R}_{+}$. Furthermore, the Borel transformation has singularities only along $\mathbb{R}_{-}$for $b \in \mathbb{R}$ including all the nonperturbative corrections.

\section{F. Dyonic loop}

The supersymmetric dyonic loop can be computed by putting a SUSY Wilson loop on $S_{b}^{1}$ [26] in the setup of the 't Hooft loop. If we assume the conjectural expression (9) for the 't Hooft loop, then the exact result for the dyonic loop should be given by

$$
D=\left\langle\operatorname{tr}_{R} e^{b a}\right\rangle_{M M},
$$

where $\langle\cdots\rangle_{M M}$ denotes the unnormalized expectation value in the matrix model (9). Since this is just an insertion of a function of $a$ without singularities, we can still apply the above technique for the 't Hooft loops to this case as well. Then, the Borel transformation is simply given by $f^{(k, k, v)}$ in Eq. (24) with the extra insertion of $\operatorname{tr}_{R} e^{b a}$ to the integrand. Thus, the analytic properties of $h^{(k, k, v)}$ do not change, and the perturbative series is still Borel summable along $\mathbb{R}_{+}$ including the instantons and the monopole bubbling effects.

\section{CONCLUSION AND DISCUSSIONS}

In this paper, we have studied weak coupling perturbative series in the presence of monopole bubbling effects and instanton effects. We have shown that the perturbative series of the 't Hooft loop in the $4 \mathrm{~d} \mathcal{N}=2$ supersymmetric gauge theories are Borel summable along $\mathbb{R}_{+}$. It has turned out that the exact result is the same as the sum of the Borel resummations over instanton-anti-instanton numbers $(k, \bar{k})$ and screened magnetic charges $v$. Our result is also nontrivially consistent with the conjecture that $4 \mathrm{~d} \mathcal{N}=2$ theories do not have IR renormalon-type singularities $[13,15]$.

There is a confusing point on our results. According to Lipatov's argument [39] and topological selection rule $[13,40]$ (see also [41]), it is expected that Borel singularities come from saddle points with the same topological number. Hence, one may expect that there are Borel singularities coming from instantons and anti-instantons with the same $k-\bar{k}$ in our setup. However, we have seen that we do not have such singularities. The absence of this type of singularities as well as IR renormalons leads the Borel summability along $\mathbb{R}_{+}$and makes the perturbative series in every sector isolated in contrast to the usual resurgence scenario in quantum mechanics [42] and QFT [13,33, $40,43]$. It would be interesting to find physical interpretations for this point. One of the possible scenarios would be "Cheshire cat resurgence," which has recently appeared in some SUSY theories [44]. Another possibility is that there may be something like a generalization of the topological selection rule particular for our setup.

We have found that the Borel transformation has infinitely many singularities in the Borel plane. It would be interesting to find their physical interpretations. Technically, these singularities come from singularities of the one-loop determinant, Nekrasov partition functions, and monopole bubbling effects. At least for those from the one-loop determinant, we expect that they can be explained by complexified SUSY solutions as in $3 \mathrm{~d} \mathcal{N}=2$ case $[33,45]$, which formally satisfy SUSY conditions but may not be on the original path integral contour.

The formula for the 't Hooft loop, which we have used, has not been explicitly derived by the supersymmetry 
localization despite it having passed the nontrivial checks. It would be nice if one could directly derive the localization formula.

It is known that the 't Hooft loop operator is $S$-dual to the SUSY Wilson loop, whose perturbative expansion is also Borel summable along $\mathbb{R}_{+}$[9]. It would be illuminating to study whether there are implications of the $S$-duality for structures of Borel singularities.

\section{ACKNOWLEDGMENTS}

We thank Takuya Okuda for his correspondence to our questions on Ref. [24]. M. H. thanks Centro de Ciencias de Benasque, CERN, Fudan University, KITP, RIKEN, and YITP for hospitality. D. Y. thanks Tokyo Institute of Technology for hospitality. This research was supported in part by the National Science Foundation under Grant No. NSF PHY-1125915. D. Y. was supported by the ERC Starting Grant No. 304806, "The Gauge/Gravity Duality and Geometry in String Theory."

\section{APPENDIX A: ANALYTIC PROPERTY OF ONE-LOOP DETERMINANT}

We discuss details on analytic properties of the one-loop determinant $Z_{1 \text { loop }}^{(v)}(a)$ as a function of $a$.

\section{Hypermultiplet contribution}

We study an analytic property of a contribution from a weight vector $\rho$ :

$$
\frac{\prod_{k=0}^{|\rho \cdot v|-1} \cosh ^{1 / 2}\left[\pi b\left(\rho \cdot a+i b\left(k-\frac{|\rho \cdot v|-1}{2}\right)\right)\right]}{\left|\Upsilon\left(i \rho \cdot a_{N}+\frac{Q}{2}\right)\right|} .
$$

From the infinite product representation (12) of $\Upsilon(x)$, we derive the following convenient identity:

$$
\begin{aligned}
\left|\Upsilon\left(i\left(x+\frac{i b v}{2}\right)+\frac{Q}{2}\right)\right|^{2} \\
=\prod_{k=0}^{|v|-1} \prod_{m_{2} \geq 0}\left[\left(m_{2}+\frac{1}{2}\right)^{2} b^{-2}+\left(x+i b\left(k-\frac{|v|-1}{2}\right)\right)^{2}\right] \\
\quad \times \prod_{m_{1}, m_{2} \geq 0}\left[\left(\left(m_{1}+\frac{|v|+1}{2}\right) b+\left(m_{2}+\frac{1}{2}\right) b^{-1}\right)^{2}+x^{2}\right]^{2} .
\end{aligned}
$$

Then, recalling $\cosh (\pi x)=\prod_{n=1}^{\infty}\left(1+\frac{4 x^{2}}{(2 n-1)^{2}}\right)$, the first term cancels the equator contribution, and we find that Eq. (A1) is proportional to

$$
\prod_{m_{1}, m_{2} \geq 0} \frac{1}{\left(\left(m_{1}+\frac{|\rho \cdot v|+1}{2}\right) b+\left(m_{2}+\frac{1}{2}\right) b^{-1}\right)^{2}+(\rho \cdot a)^{2}} .
$$

Thus, we do not have branch cuts but have simple poles at

$\rho \cdot a= \pm i\left[\left(m_{1}+\frac{|\rho \cdot v|+1}{2}\right) b+\left(m_{2}+\frac{1}{2}\right) b^{-1}\right]$.

\section{Vector multiplet contribution}

Next, we study an analytic property of each positive root contribution:

$$
\frac{\left|\Upsilon\left(i \alpha \cdot a_{N}\right) \Upsilon\left(-i \alpha \cdot a_{N}\right)\right|}{\prod_{ \pm} \prod_{k=0}^{|\alpha \cdot v|-1} \sinh ^{1 / 2}\left[\pi b\left( \pm \alpha \cdot a+i b\left(k-\frac{|\alpha \cdot v|}{2}\right)\right)\right]} .
$$

For this case, it is convenient to use the identity

$$
\begin{aligned}
& \left|\Upsilon\left(i\left(x+\frac{i b v}{2}\right)\right) \Upsilon\left(-i\left(x+\frac{i b v}{2}\right)\right)\right|^{2} \\
& \propto \prod_{ \pm} \prod_{k=0}^{|v|-1} \sinh \left[\pi b\left( \pm x+i b\left(k-\frac{|v|-1}{2}\right)\right)\right] \\
& \quad \times \prod_{m_{1}, m_{2} \geq 0}\left[\left(\left(m_{1}+\frac{|v|+1}{2}\right) b+\left(m_{2}+\frac{1}{2}\right) b^{-1}\right)^{2}\right. \\
& \left.\quad+\left( \pm x+\frac{i Q}{2}\right)^{2}\right]^{2} .
\end{aligned}
$$

Then, the first term cancels the equator contribution, and Eq. (A5) becomes proportional to

$$
\begin{aligned}
& \prod_{m_{1}, m_{2} \geq 0}\left[\left(\left(m_{1}+\frac{|\alpha \cdot v|+1}{2}\right) b+\left(m_{2}+\frac{1}{2}\right) b^{-1}\right)^{2}\right. \\
& \left.+\left(\alpha \cdot a+\frac{i Q}{2}\right)^{2}\right], \quad \text { with } \quad \alpha \in \Delta .
\end{aligned}
$$

Thus, the vector one-loop determinant does not have any singularity. Note also that this has simple zeros at

$\alpha \cdot a=-\frac{i Q}{2} \pm i\left[\left(m_{1}+\frac{|\alpha \cdot v|+1}{2}\right) b+\left(m_{2}+\frac{1}{2}\right) b^{-1}\right]$.

Therefore, the whole one-loop determinant does not have a branch cut and is a meromorphic function of $a$ whose poles are not located on the real axis. This point is directly connected to Borel summability along $\mathbb{R}_{+}$. 


\section{APPENDIX B: DETAILS ON MONOPOLE BUBBLING EFFECTS}

We explain details of the monopole bubbling effect (31). As we mentioned, the sum over $Y$ in Eq. (31) and the product over $s$ in Eq. (32) are constrained. $Y$ in Eq. (31) is a set of Young diagrams $\left(Y=\left\{Y_{1}, \ldots, Y_{N}\right\}\right)$, and the sum is over all possible configurations of $p$ boxes distributed to $Y$. The number of the boxes $p$ is determined by the dimension of a vector $K=\left(K_{1}, \ldots, K_{p}\right)$, which is specified by the following equation [25]:

$$
\operatorname{tr}\left(\alpha^{B}\right)=\operatorname{tr}\left(\alpha^{v}\right)+\alpha^{-1}(\alpha-1)^{2} \operatorname{tr}\left(\alpha^{K}\right),
$$

where $\alpha$ is an arbitrary element of $U(1)$ and the trace is the sum over all components of the vector. Taking $\alpha=e^{i \epsilon}$ and comparing $\mathcal{O}\left(\epsilon^{2}\right)$, this condition uniquely determines $p$ as $p=\frac{1}{2} \operatorname{tr}\left(B^{2}-v^{2}\right)$. Especially when $v=B$, we do not have solutions, and, therefore, the monopole bubbling effect is trivial as mentioned in Eq. (14). The products in terms of $i$, $j, s$ in Eq. (32) have two constraints. One is

$$
v_{i(s)}+s_{h}-s_{v} \in\left\{K_{t}\right\}_{t=1}^{p},
$$

where $i(s)$ is a gauge group index of Young tableau to which $s$ belongs. The other constraint depends on the representation. The constraint for vector and adjoint representations is

$$
v_{i j}+L_{Y_{j}}(s)+A_{Y_{i}}(s)+1=0,
$$

while the one for fundamental representations is

$$
v_{i(s)}-s_{v}+s_{h}=0 .
$$

In order to find $(B, v)$ for $S U(N)$, it is convenient to start with the vectors $(\tilde{B}, \tilde{v})$ satisfying the above constraints for $U(N)$ and impose the traceless condition:

$$
B=\tilde{B}-\frac{1}{N} \sum_{i=1}^{N} \tilde{B}_{i}, \quad v=\tilde{v}-\frac{1}{N} \sum_{i=1}^{N} \tilde{v}_{i} .
$$

[1] A. Kapustin and E. Witten, Electric-magnetic duality and the geometric Langlands program, Commun. Numer. Theor. Phys. 1, 1 (2007).

[2] G. 't Hooft, Magnetic monopoles in unified gauge theories, Nucl. Phys. B79, 276 (1974); A. M. Polyakov, Particle spectrum in the quantum field theory, Pis'ma Zh. Eksp. Teor. Fiz. 20, 430 (1974) [JETP Lett. 20, 194 (1974)].

[3] See Ref. [4] for an explicit example.

[4] S. A. Cherkis and B. Durcan, The 't Hooft-Polyakov monopole in the presence of an 't Hooft operator, Phys. Lett. B 671, 123 (2009).

[5] G. 't Hooft, On the phase transition towards permanent quark confinement, Nucl. Phys. B138, 1 (1978).

[6] K. G. Wilson, Confinement of quarks, Phys. Rev. D 10, 2445 (1974).

[7] A. Kapustin, Wilson-'t Hooft operators in four-dimensional gauge theories and S-duality, Phys. Rev. D 74, 025005 (2006).

[8] F. J. Dyson, Divergence of perturbation theory in quantum electrodynamics, Phys. Rev. 85, 631 (1952).

[9] M. Honda, Borel Summability of Perturbative Series in 4D $N=2$ and $5 \mathrm{D} N=1$ Supersymmetric Theories, Phys. Rev. Lett. 116, 211601 (2016).

[10] See Refs. [11,12] for earlier checks in some examples.

[11] J. G. Russo, A note on perturbation series in supersymmetric gauge theories, J. High Energy Phys. 06 (2012) 038.

[12] I. Aniceto, J. G. Russo, and R. Schiappa, Resurgent analysis of localizable observables in supersymmetric gauge theories, J. High Energy Phys. 03 (2015) 172; E. Gerchkovitz, J. Gomis, N. Ishtiaque, A. Karasik, Z. Komargodski, and
S. S. Pufu, Correlation functions of Coulomb branch operators, J. High Energy Phys. 01 (2017) 103.

[13] P. Argyres and M. Unsal, A Semiclassical Realization of Infrared Renormalons, Phys. Rev. Lett. 109, 121601 (2012); The semi-classical expansion and resurgence in gauge theories: New perturbative, instanton, bion, and renormalon effects, J. High Energy Phys. 08 (2012) 063.

[14] G. 't Hooft, Can we make sense out of quantum chromodynamics?, Subnuclear series 15, 943 (1979).

[15] E. Poppitz and M. Unsal, Seiberg-Witten and 'Polyakov-like' magnetic bion confinements are continuously connected, J. High Energy Phys. 07 (2011) 082.

[16] G. V. Dunne, M. Shifman, and M. Unsal, Infrared Renormalons versus Operator Product Expansions in Supersymmetric and Related Gauge Theories, Phys. Rev. Lett. 114, 191601 (2015).

[17] J. Gomis, T. Okuda, and D. Trancanelli, Quantum 't Hooft operators and S-duality in $N=4$ super Yang-Mills, Adv. Theor. Math. Phys. 13, 1941 (2009).

[18] Note that, because of $|v| \leq|B|$, the contributions from the sector $|v| \neq|B|$ are relatively exponentially suppressed corrections.

[19] Reference [11] studied the 't Hooft loop in $S U(2) \mathcal{N}=2^{*}$ Yang-Mills theory on round $S^{4}$ in the perturbative sector.

[20] V. Pestun, Localization of gauge theory on a four-sphere and supersymmetric Wilson loops, Commun. Math. Phys. 313, 71 (2012).

[21] M. Marino, Semiclassical decoding, in Proceedings at KITP, 2017, http://online.kitp.ucsb.edu/online/resurgent_c17/ marino. 
[22] Y. Ito, T. Okuda, and M. Taki, Line operators on $S^{1} \times R^{3}$ and quantization of the Hitchin moduli space, J. High Energy Phys. 04 (2012) 010; Erratum, J. High Energy Phys. 03 (2016) 85.

[23] T. Okuda, Line operators in supersymmetric gauge theories and the $2 \mathrm{~d}-4 \mathrm{~d}$ relation, in New Dualities of Supersymmetric Gauge Theories, edited by J. Teschner (Springer, 2016), pp. 195-222.

[24] J. Gomis, T. Okuda, and V. Pestun, Exact results for 't Hooft loops in gauge theories on $S^{4}$, J. High Energy Phys. 05 (2012) 141.

[25] D. Gang, E. Koh, and K. Lee, Line operator index on $S^{1} \times S^{3}$, J. High Energy Phys. 05 (2012) 007.

[26] N. Hama and K. Hosomichi, Seiberg-Witten theories on ellipsoids, J. High Energy Phys. 09 (2012) 033.

[27] T. Nosaka and S. Terashima, Supersymmetric gauge theories on a squashed four-sphere, J. High Energy Phys. 12 (2013) 001; A. Cabo-Bizet, E. Gava, V. I. Giraldo-Rivera, M. N. Muteeb, and K. S. Narain, Partition function of $N=2$ gauge theories on a squashed $S^{4}$ with $S U(2) \times U(1)$ isometry, Nucl. Phys. B899, 149 (2015).

[28] L. F. Alday, D. Gaiotto, and Y. Tachikawa, Liouville correlation functions from four-dimensional gauge theories, Lett. Math. Phys. 91, 167 (2010).

[29] We take $r=1$. We can recover $r$ dependence by taking $a \rightarrow r a$.

[30] Note that $\alpha \cdot v$ and $\rho \cdot v$ are integers by Dirac quantization conditions [31].

[31] P. Goddard, J. Nuyts, and D. I. Olive, Gauge theories and magnetic charge, Nucl. Phys. B125, 1 (1977).

[32] N. A. Nekrasov, Seiberg-Witten prepotential from instanton counting, Adv. Theor. Math. Phys. 7, 831 (2003); N. Nekrasov and A. Okounkov, Seiberg-Witten theory and random partitions, Progress of mathematics 244, 525 (2006).

[33] M. Honda, How to resum perturbative series in $3 \mathrm{~d} \mathrm{~N}=2$ Chern-Simons matter theories, Phys. Rev. D 94, 025039 (2016).

[34] Explicitly, $\sharp=\frac{N-2}{2}+\sum_{i<j} \delta_{v_{i j}, 0}$.

[35] For example, it is convenient to make the replacements $\pm \hat{x}_{i} \rightarrow 1, \pm \hat{x}_{i j} \rightarrow 2, \zeta(\ell>1) \rightarrow 2$, and $1 /\left[\left(m_{1} b+m_{2} b^{-1}\right)^{2}+\right.$ $\left.x^{2}\right] \rightarrow 1 /\left[b^{ \pm 2}\left(m_{1}+m_{2}\right)^{2}+x^{2}\right]$.

[36] If $G_{p}$ is $S U$, then we insert the traceless constraint by the delta function into $h^{(k, \bar{k}, v)}$.

[37] $(k, \bar{k}, v)$ stands for $k=\left\{k_{1}, \ldots, k_{p}\right\}, \bar{k}=\left\{\bar{k}_{1}, \ldots, \bar{k}_{p}\right\}$, and $v=\left\{v^{(1)}, \ldots, v^{(p)}\right\}$.

[38] Explicitly, $\sharp_{p}=\frac{\left|G_{p}\right|-1}{2}+\sum_{\alpha_{p} \in \Delta_{+}^{(p)}} \delta_{\alpha_{p} \cdot v^{(p)}, 0}$.

[39] L. N. Lipatov, Divergence of the perturbation theory series and the quasiclassical theory, Zh. Eksp. Teor. Fiz. 72, 411 (1977) [Sov. Phys. JETP 45, 216 (1977)].

[40] G. V. Dunne and M. Unsal, Resurgence and trans-series in quantum field theory: The $\mathrm{CP}(\mathrm{N}-1)$ model, J. High Energy Phys. 11 (2012) 170; Continuity and resurgence: Towards a continuum definition of the $\mathbb{C P}(N-1)$ model, Phys. Rev. D 87, 025015 (2013).

[41] I. Aniceto and R. Schiappa, Nonperturbative ambiguities and the reality of resurgent transseries, Commun. Math. Phys. 335, 183 (2015).

[42] E. B. Bogomolny, Calculation of instanton-anti-instanton contributions in quantum mechanics, Phys. Lett. 91B, 431 (1980); J. Zinn-Justin, Multi-instanton contributions in quantum mechanics, Nucl. Phys. B192, 125 (1981); G. V. Dunne and M. Unsal, Generating nonperturbative physics from perturbation theory, Phys. Rev. D 89, 041701 (2014); Uniform WKB, multi-instantons, and resurgent trans-series, Phys. Rev. D 89, 105009 (2014); WKB and resurgence in the Mathieu equation, arXiv:1603.04924; G. Basar, G. V. Dunne, and M. Unsal, Resurgence theory, ghost-instantons, and analytic continuation of path integrals, J. High Energy Phys. 10 (2013) 041; Quantum geometry of resurgent perturbative/nonperturbative relations, J. High Energy Phys. 05 (2017) 087; T. Misumi, M. Nitta, and N. Sakai, Resurgence in sine-Gordon quantum mechanics: Exact agreement between multi-instantons and uniform WKB, J. High Energy Phys. 09 (2015) 157; A. Behtash, E. Poppitz, T. Sulejmanpasic, and M. Unsal, The curious incident of multi-instantons and the necessity of Lefschetz thimbles, J. High Energy Phys. 11 (2015) 175; A. Behtash, G. V. Dunne, T. Schaefer, T. Sulejmanpasic, and M. Unsal, Toward Picard-Lefschetz theory of path integrals, complex saddles and resurgence, arXiv:1510.03435; T. Fujimori, S. Kamata, T. Misumi, M. Nitta, and N. Sakai, Nonperturbative contributions from complexified solutions in $\mathbb{C} \mathbb{P}^{N-1}$ models, Phys. Rev. D 94, 105002 (2016); Exact resurgent transseries and multibion contributions to all orders, Phys. Rev. D 95, 105001 (2017); Resurgence structure to all orders of multi-bions in deformed SUSY quantum mechanics, Prog. Theor. Exp. Phys. 2017, 083B02 (2017).

[43] A. Cherman, D. Dorigoni, G. V. Dunne, and M. Unsal, Resurgence in Quantum Field Theory: Nonperturbative Effects in the Principal Chiral Model, Phys. Rev. Lett. 112, 021601 (2014); A. Cherman, D. Dorigoni, and M. Unsal, Decoding perturbation theory using resurgence: Stokes phenomena, new saddle points and Lefschetz thimbles, J. High Energy Phys. 10 (2015) 056; T. Misumi, M. Nitta, and N. Sakai, Neutral bions in the $\mathbb{C P}^{N-1}$ model, J. High Energy Phys. 06 (2014) 164; G. V. Dunne and M. Unsal, Resurgence and dynamics of $\mathrm{O}(\mathrm{N})$ and Grassmannian sigma models, J. High Energy Phys. 09 (2015) 199; P. V. Buividovich, G. V. Dunne, and S. N. Valgushev, Complex Path Integrals and Saddles in Two-Dimensional Gauge Theory, Phys. Rev. Lett. 116, 132001 (2016); S. Demulder, D. Dorigoni, and D. C. Thompson, Resurgence in $\eta$-deformed principal chiral models, J. High Energy Phys. 07 (2016) 088; S. Gukov, M. Marino, and P. Putrov, Resurgence in complex Chern-Simons theory, arXiv:1605.07615; A. Ahmed and G. V. Dunne, Transmutation of a trans-series: The GrossWitten-Wadia phase transition, J. High Energy Phys. 11 (2017) 054; D. Gang and Y. Hatsuda, S-duality resurgence in SL(2) Chern-Simons theory, J. High Energy Phys. 07 (2018) 053.

[44] C. Kozcaz, T. Sulejmanpasic, Y. Tanizaki, and M. Unsal, Cheshire cat resurgence, self-resurgence and quasi-exact solvable systems, Commun. Math. Phys. 364, 835 (2018); G. V. Dunne and M. Unsal, Deconstructing zero: Resurgence, supersymmetry and complex saddles, J. High Energy Phys. 12 (2016) 002; D. Dorigoni and P. Glass, The grin of Cheshire cat resurgence from supersymmetric localization, SciPost Phys. 4, 012 (2018).

[45] M. Honda, Supersymmetric Solutions and Borel Singularities for $N=2$ Supersymmetric Chern-Simons Theories, Phys. Rev. Lett. 121, 021601 (2018). 